\section{Fatores associados à retenção de peso pós- parto em uma coorte de mulheres, 2005-2007}

Fernanda Rebelo 1

Maria Beatriz T. Castro 2

Carmem L. Dutra 3

Michael M. Schlussel 4

Gilberto Kac 5

\section{Factors associated with post-partum weight retention in a cohort of women, 2005-2007}

\begin{abstract}
Objective: to investigate factors associated with post-partum weight retention.

Methods: a cohort study with 104 recruited pregnant women in the $8^{\text {th }}$ to $13^{\text {th }}$ week of gestation was carried out at Basic Health Unit in the municipality of Rio de Janeiro and the women were accompanied until around 55 days post-partum. The variables included socio-demographic, economic, biochemical, anthropometric and reproductive data. Post-partum weight retention (the difference between post partum and pre-gestational weight) was used as the dependent variable.

Results: the mean weight retention was $3.2 \mathrm{~kg} \pm$ $3.7 \mathrm{~kg}$. Women with excessive gestational weight gain retained $5.0 \mathrm{~kg} \pm 3.9$ in comparison to $3.6 \mathrm{~kg} \pm 3.0 \mathrm{in}$ women with adequate gestational weight gain and 1.4 $\mathrm{kg} \pm 3.1$ compared to those with inadequate gestational weight gain. The gestational weight gain and pre-gestational body mass index continued to show a significant association with weight retention in the final model. It was found that for every kilogram of weight gain during gestation, almost $50 \%$ was retained post-partum $(\beta=0.494 ; p<0.001)$ and every $1.0 \mathrm{~kg} / \mathrm{m}^{2}$ less in pre-gestational BMI corresponded to a retention of approximately $150 \mathrm{~g}(\beta=-0.149$; $p<0.05)$.

Conclusion: gestational weight gain is positively associated with post-partum weight retention, while pre-gestational BMI is inversely associated with the dependent variable. Nutritional advice on weight control may help minimize the incidence of obesity in women during post-partum.
\end{abstract}

Key words Weight gain, Pregnancy, Prospective studies, Body mass index
1-5 Instituto de Nutrição Josué de Castro. Universidade Federal do Rio de Janeiro. Av. Brigadeiro Trompowsky, s.n. CCS, Bloco J, $2^{\circ}$ andar, sala 29. Cidade Universitária - Ilha do Fundão. Rio de Janeiro, RJ, Brasil. CEP: 21941-590.

E-mail: gkac@nutricao.ufrj.br

\section{Resumo}

Objetivo: investigar fatores associados à retenção de peso pós-parto.

Métodos: estudo de coorte com 104 gestantes recrutadas em uma Unidade Básica de Saúde no município do Rio de Janeiro, da $8^{a}$ a $13^{a}$ semana de gestação e acompanhadas até aproximadamente 55 dias pós-parto. As variáveis incluiram informações sócio-demográficas, econômicas, bioquímicas, antropométricas e reprodutivas. A retenção de peso pós-parto (diferença entre o peso pós-parto e o peso pré-gestacional) foi utilizada como variável dependente.

Resultados: a média de peso retido foi $3,2 \mathrm{~kg} \pm$ $3,7 \mathrm{~kg}$. As mulheres com ganho de peso gestacional (GPG) excessivo retiveram $5,0 \mathrm{~kg} \pm 3,9 \mathrm{em}$ comparação a 3,6 kg $\pm 3,0$ em mulheres com $G P G$ adequado e 1,4 $\mathrm{kg} \pm 3,1$ para as com GPG insuficiente. O GPG e o Índice de Massa corporal (IMC) pré-gestacional mantiveram associação significativa com a retenção de peso no modelo final. Observou-se que a cada quilo de peso ganho na gestação, quase $50 \%$ ficaram retidos no pós-parto $(\beta=0,494$; $p<0,001)$ e a cada $1,0 \mathrm{~kg} / \mathrm{m}^{2}$ a menos no IMC prégestacional correspondeu a uma retenção de aproximadamente $150 \mathrm{~g}(\beta=-0,149 ; p<0,05)$.

Conclusão: o GPG está positivamente e o IMC pré-gestacional inversamente associado à retenção de peso pós-parto. Orientações nutricionais sobre o controle do ganho ponderal podem ajudar a minimizar a incidência de obesidade entre mulheres no pós-parto.

Palavras-chave Ganho de peso, Gravidez, Estudos prospectivos, Índice de massa corporal 


\section{Introdução}

Sabe-se que a obesidade é um problema de saúde pública em diversos países. ${ }^{1,2}$ Desta forma é importante identificar os grupos populacionais mais susceptíveis, os momentos e as estratégias adequadas para a prevenção do ganho de peso excessivo. Diversos estudos revelam que as mulheres durante o período reprodutivo estão mais susceptíveis ao ganho de peso excessivo e à manutenção ou desenvolvimento da obesidade, especialmente devido à retenção de peso pós-parto. ${ }^{2-4}$

Apesar de ainda não haver um ponto de corte a partir do qual a retenção de peso pós-parto seja considerada significativa, alguns estudos já demonstraram um importante aumento ponderal após a gestação.5,6 Huang et al.2 observaram que a frequência de sobrepeso/obesidade aumentou de $18,3 \%$ antes da gravidez para $27,6 \%$ seis meses pósparto em mulheres Taiwanesas. Em um estudo de coorte que acompanhou mulheres até 15 anos pósparto, Linné et al.7 mostraram que a retenção de peso um ano após o parto pôde predizer o sobrepeso 15 anos depois da gestação. A retenção de peso pósparto consiste, portanto, em um importante indicador nutricional a ser monitorado. 4,7

Os principais fatores associados à retenção de peso pós-parto são: o ganho de peso gestacional excessivo, o estado nutricional pré-gestacional, amamentação, raça, idade, paridade, estado civil, atividade física e consumo alimentar. 8 Neste sentido, o presente estudo apresentou como objetivo avaliar a associação entre variáveis socioeconômicas, reprodutivas, antropométricas e bioquímicas e a retenção de peso pós-parto em mulheres atendidas em uma unidade de atenção básica do Sistema Único de Saúde (SUS) na cidade do Rio de Janeiro, Brasil.

\section{Métodos}

Este estudo consiste em uma coorte prospectiva de gestantes. O recrutamento das mulheres participantes do estudo ocorreu no período entre junho de 2005 e abril de 2007. O monitoramento contou com quatro ondas de seguimento durante a gestação: $8^{\mathrm{a}}$ $13^{\mathrm{a}}$ (baseline), 19a $-21^{\mathrm{a}}, 26^{\mathrm{a}}-28^{\mathrm{a}}, 36^{\mathrm{a}}-40^{\mathrm{a}}$ semana gestacional; e com uma onda de seguimento programada para ocorrer com no mínimo 30 dias pós-parto. Nas entrevistas foram coletados dados socioeconômicos, dietéticos, bioquímicos, antropométricos, variáveis reprodutivas pregressas e do curso da gestação. Com exceção da primeira entrevista, as demais foram realizadas em dias previamente agendados, na Unidade Básica de Saúde
Madre Teresa de Calcutá, da Secretaria Municipal de Saúde (SMS) do Município do Rio de Janeiro, onde também ocorreu a captação das gestantes.

As mulheres foram recrutadas obedecendo aos seguintes critérios de elegibilidade: máximo de 13 semanas de gestação, calculada por meio da data da última menstruação (DUM) informada pela mulher; idade cronológica entre 18 e 40 anos; não possuir doenças crônicas como hipertensão arterial e diabetes, nem doenças infecto-parasitárias, como infecção por HIV (vírus da imunodeficiência adquirida humana); não apresentar gestação gemelar; e residir na área adstrita ao local do estudo.

A retenção de peso pós-parto, variável desfecho, foi calculada a partir da subtração do peso medido na $5^{\mathrm{a}}$ onda de seguimento pelo peso pré-gestacional, aferido na $1^{\mathrm{a}}$ onda de seguimento (baseline). A categorização das variáveis independentes foi baseada no teste prévio de múltiplos pontos de corte, sendo escolhidos aqueles que apresentaram melhor distribuição e mostraram melhores associações com a retenção de peso pós-parto, assim como aqueles já descritos na literatura e que mantiveram plausibilidade biológica com o desfecho estudado.

As seguintes variáveis independentes foram incluídas nas análises: a) variáveis sócio-demográficas e econômicas: idade (18-24; 25-40 anos); estado marital (casada; vive em união; não vive em união;), cor da pele autoclassificada (branca; parda; negra), tabagismo (fumante; ex-fumante; não fumante), renda familiar per capita $(<300,00 ; \geq$ 300,00 reais), escolaridade (0-8; 9-16 anos); b) variáveis antropométricas: estatura $(1,43-1,59 ; 1,60$ $1,76 \mathrm{~m}$ ); IMC pré-gestacional segundo o Institute of Medicine (IOM), 20099 (baixo peso $<18,5 \mathrm{~kg} / \mathrm{m}^{2}$; eutrofia $18,5-24,9 \mathrm{~kg} / \mathrm{m}^{2}$; sobrepeso $25,0-29,9 \mathrm{~kg} / \mathrm{m}^{2}$ ou obesidade $\left.\geq 30,0 \mathrm{~kg} / \mathrm{m}^{2}\right)$; adequação do ganho de peso gestacional total (insuficiente; adequado ou excessivo); peso da criança ao nascer (1250-3500; 3501-4800 g); c) variáveis reprodutivas: tipo de parto (normal; cesárea); amamentação exclusiva (sim, não); idade da menarca (8-12; 13-18 anos); paridade $(0 ; \geq 1$ partos); d) variáveis bioquímicas: glicemia de jejum (tercis, $\mathrm{mg} / \mathrm{dL}$ ), colesterol total (tercis, mg/dL), leptina sérica (tercis, ng/dL).

As mulheres foram pesadas em balança com escala digital (Filizzola Ltda.) e a estatura aferida em duplicata com estadiômetro portátil (Harpenden Inc.). Todas as medidas antropométricas foram padronizadas segundo recomendação de Gordon et al. 10 e realizadas por entrevistadores treinados.

$\mathrm{O}$ índice de massa corporal (IMC) pré-gestacional [peso $(\mathrm{kg}) /$ estatura $(\mathrm{m})^{2}$ ] foi calculado com base nos dados medidos durante a primeira onda de 
seguimento, período máximo para definição do estado nutricional pré-gestacional. Foram considerados os novos pontos de corte propostos pelo IOM para a classificação do estado nutricional pré-gestacional das participantes. ${ }^{9}$ Para o cálculo do ganho de peso gestacional total realizou-se a subtração das medidas de peso corporal aferidas na quarta e na primeira onda de seguimento. A adequação do ganho de peso gestacional foi avaliada segundo as novas recomendações do IOM baseadas no IMC pré-gestacional. Assim, mulheres com baixo peso deveriam ganhar entre 12,5 e $18 \mathrm{~kg}$; mulheres com IMC adequado entre 11,5 e $16 \mathrm{~kg}$, mulheres com sobrepeso entre 7 e 11,5 kg e mulheres com obesidade entre 5 e 9 kg. ${ }^{9}$ Valores abaixo ou acima do recomendando pelo IOM foram considerados ganho de peso gestacional insuficiente e excessivo, respectivamente.

A análise bioquímica do sangue das gestantes foi realizada a partir de amostras coletadas por profissional da unidade básica de saúde, capacitado para esta função, no primeiro trimestre de gestação (aproximadamente 9,8 semanas de idade gestacional). As mulheres cumpriram jejum de no mínimo 12 horas. As amostras foram armazenadas em laboratório com condições e temperatura padrão. A dosagem da concentração de leptina sérica foi realizada em duplicata (mesma amostra, mesma placa, poços diferentes) por meio de ensaio imunoenzimático (ELISA) utilizando-se kits comerciais (Linco Research, St. Charles, Missouri) e seguindo as instruções sugeridas pelo fabricante. Para determinação do colesterol total e da glicemia empregouse o método de colorimetria enzimática, realizado em laboratório de referência da Secretária Municipal de Saúde do Rio de Janeiro.

Inicialmente foram calculadas as médias e seus respectivos desvios padrão da retenção de peso segundo cada variável independente. Para tais análises foram empregados os seguintes testes: teste t-Student e análise de variância (ANOVA). Posteriormente empregou-se regressão linear simples para cada variável independente tendo como variável dependente a retenção de peso pós-parto. Todas as variáveis que apresentaram valor de $p \leq 0,20$ nessa primeira fase foram inicialmente incluídas no modelo de regressão linear multivariada. As variáveis cujo valor de $p$ foi $<0,05$ permaneceram no modelo final, no qual o tempo pós-parto (dias) foi utilizado como variável controle. Todas as análises foram realizadas utilizando o Statistical Package for Social Science versão 16.0 (SPSS 16.0).

Este estudo foi aprovado pelo Comitê de Ética em Pesquisa (CEP) do Instituto de Puericultura e Pediatria Martagão Gesteira (IPPMG), da Universidade Federal do Rio de Janeiro.

\section{Resultados}

Entre as 255 gestantes avaliadas na coorte, 104 $(40,8 \%)$ completaram o seguimento e, portanto, possuíam dados sobre a retenção de peso pós-parto. Não houve diferença significativa entre as mulheres excluídas e as que permaneceram nas análises, exceto para estado marital (Tabela 1).

As mulheres investigadas tinham em média 25,7 $( \pm 5,6)$ anos, 9,2 $( \pm 2,9)$ anos de escolaridade, IMC pré-gestacional médio de $24,0 \mathrm{~kg} / \mathrm{m}^{2}( \pm 4,5)$ e ganho de peso gestacional médio de $12,2 \mathrm{~kg}( \pm 8,3)$. A média da retenção de peso pós-parto foi de $3,20 \mathrm{~kg}$ $( \pm 3,7)$, variando de $-6,0 \mathrm{~kg}$ a $12,1 \mathrm{~kg}$.

Verificou-se que as mulheres mais jovens (18-24 anos) apresentaram maior retenção de peso pós-parto $(3,9 \mathrm{~kg} \pm 4,0)$ quando comparadas àquelas com 25$40 \operatorname{anos}(2,3 \mathrm{~kg} \pm 3,0)$. A retenção de peso foi maior entre as mulheres que apresentaram ganho de peso gestacional excessivo $(5,0 \mathrm{~kg} \pm 3,9)$, em comparação às mulheres com ganho de peso gestacional adequado $(3,6 \mathrm{~kg} \pm 3,0)$ ou insuficiente $(1,4 \mathrm{~kg} \pm$ $3,1)$, como se pode observar na Tabela 2 .

Não houve diferença significativa para a retenção de peso pós-parto segundo as variáveis reprodutivas e bioquímicas (Tabela 3 ).

As análises de regressão linear simples demonstraram que o ganho de peso gestacional foi a variável com maior força de associação com a variável retenção de peso pós-parto. A associação entre idade e retenção de peso pós-parto foi negativa, porém limítrofe no que diz respeito à significância estatística. Para cada ano a mais de idade, a retenção de peso foi $115 \mathrm{~g}$ menor (Tabela 4 ).

As variáveis incluídas no modelo multivariado foram: tipo de parto, estado marital, colesterol total, tabagismo, IMC pré-gestacional, idade e ganho de peso gestacional total. No modelo final de regressão linear multivariado, apenas o ganho de peso gestacional total $(p<0,001)$ e o IMC pré-gestacional $(p<0,05)$ mantiveram associação significativa com a retenção de peso pós-parto e, juntos, explicam $40,7 \%$ da variabilidade do desfecho no grupo estudado. Observou-se que para cada quilo de peso ganho na gestação, houve retenção pós-parto de quase $50 \%(\beta=0,494 ; p<0,001)$ do ganho e para cada $1,0 \mathrm{~kg} / \mathrm{m}^{2}$ a menos no IMC pré-gestacional houve retenção de aproximadamente $150 \mathrm{~g}(\beta=$ $0,149 ; p<0,05)$ (Tabela 5). 
Tabela 1

Análise das perdas de seguimento. Rio de Janeiro, 2005 - 2007.

\begin{tabular}{|c|c|c|c|c|c|}
\hline Variáveis & $\begin{array}{l}\text { Observações } \\
\text { iniciais }\end{array}$ & $\begin{array}{c}\text { Perdas de } \\
\text { seguimento }\end{array}$ & $\begin{array}{l}\text { Seguimento } \\
\text { completo }\end{array}$ & $\begin{array}{l}\text { Taxa final de } \\
\text { seguimento (\%) }\end{array}$ & $p^{1}$ \\
\hline \multicolumn{6}{|l|}{ Idade (anos) } \\
\hline $18-24$ & 143 & 84 & 59 & 41,3 & 0,862 \\
\hline $25-40$ & 112 & 67 & 45 & 40,2 & \\
\hline \multicolumn{6}{|l|}{ Estado marital } \\
\hline Casada & 55 & 35 & 20 & 36,4 & \\
\hline Vive em união & 143 & 91 & 52 & 36,4 & 0,028 \\
\hline Não vive em união & 57 & 25 & 32 & 56,1 & \\
\hline \multicolumn{6}{|l|}{ Cor da pele 2} \\
\hline Branca & 66 & 43 & 23 & 34,8 & \\
\hline Parda & 164 & 95 & 69 & 42,1 & 0,515 \\
\hline Negra & 24 & 13 & 11 & 45,8 & \\
\hline \multicolumn{6}{|l|}{ Tabagismo } \\
\hline Fumante & 31 & 24 & 7 & 22,6 & \\
\hline Ex-fumante & 63 & 38 & 25 & 39,7 & 0,070 \\
\hline Não fumante & 161 & 89 & 72 & 44,7 & \\
\hline \multicolumn{6}{|l|}{ Renda per capita (em Reais) } \\
\hline $1^{\circ}$ quartil $(<161,20)$ & 71 & 48 & 23 & 32,4 & \\
\hline $2^{\circ}$ quartil $(161,20-274,00)$ & 62 & 38 & 24 & 38,7 & 0,157 \\
\hline $3^{\circ}$ quartil $(275,00-449,00)$ & 62 & 30 & 32 & 51,6 & \\
\hline $4^{\circ}$ quartil $(\geq 450,00)$ & 60 & 35 & 25 & 41,7 & \\
\hline \multicolumn{6}{|l|}{ Escolaridade (anos) } \\
\hline $0-8$ & 121 & 78 & 43 & 35,5 & 0,105 \\
\hline $9-16$ & 134 & 73 & 61 & 45,5 & \\
\hline \multicolumn{6}{|l|}{ IMC pré-gestacional $\left(\mathrm{kg} / \mathrm{m}^{2}\right)^{3}$} \\
\hline Baixo peso $(<18,5)$ & 14 & 9 & 5 & 35,7 & \\
\hline Eutrofia $(18,5-24,9)$ & 156 & 88 & 68 & 43,6 & 0,519 \\
\hline Sobrepeso/ Obesidade $(\geq 25)$ & 85 & 54 & 31 & 36,5 & \\
\hline \multicolumn{6}{|l|}{ Adequação do ganho de peso } \\
\hline \multicolumn{6}{|l|}{ total4 } \\
\hline Insuficiente & 59 & 22 & 37 & 62,7 & \\
\hline Adequado & 61 & 28 & 33 & 54,1 & 0,630 \\
\hline Excessivo & 44 & 18 & 26 & 59,1 & \\
\hline \multicolumn{6}{|l|}{ Glicemia (mg/dL) } \\
\hline $1^{\circ}$ tercil $(<72,6)$ & 74 & 44 & 30 & 40,5 & \\
\hline $2^{\circ}$ tercil $(72,6 \leq 80,4)$ & 74 & 39 & 35 & 47,3 & 0,631 \\
\hline $3^{\circ}$ tercil $(>80,4)$ & 74 & 44 & 30 & 40,5 & \\
\hline \multicolumn{6}{|l|}{ Colesterol total $(\mathrm{mg} / \mathrm{dL})$} \\
\hline $1^{\circ}$ tercil $(<155,0)$ & 72 & 45 & 27 & 37,5 & \\
\hline $2^{\circ}$ tercil $(155,0 \leq 186,0)$ & 76 & 45 & 31 & 40,8 & 0,252 \\
\hline $3^{\circ}$ tercil $(>80,4)$ & 71 & 35 & 36 & 50,7 & \\
\hline \multicolumn{6}{|l|}{ Leptina sérica $(\mathrm{ng} / \mathrm{mL})$} \\
\hline $1^{\circ}$ tercil $(<12,9)$ & 54 & 29 & 25 & 46,3 & \\
\hline $2^{\circ}$ tercil $(12,9 \leq 28,5)$ & 54 & 31 & 23 & 42,6 & 0,742 \\
\hline $3^{\circ}$ tercil $(>28,5)$ & 54 & 27 & 27 & 50,0 & \\
\hline
\end{tabular}

$1 p$ refere-se ao teste de qui-quadrado para proporções; 2 Um dado faltante para cor de pele no baseline e com seguimento completo; 3 Índice de massa corporal classificado de acordo com o Institute of Medicine (IOM, 2009): 4 Ganho de peso gestacional classificado de acordo com o Institute of Medicine (IOM, 2009). 
Tabela 2

Média de retenção de peso pós-parto segundo as co-variáveis sócio-demográficas, econômicas e antropométricas. Rio de Janeiro, 2005-2007.

\begin{tabular}{|c|c|c|c|c|c|}
\hline Variáveis & $\mathrm{N}$ & $\%$ & $\begin{array}{l}\bar{X} \text { de retenção de peso } \\
\text { pós-parto }(\mathrm{kg})\end{array}$ & DP & $p 1$ \\
\hline \multicolumn{6}{|l|}{ Idade (anos) } \\
\hline $18-24$ & 59 & 56,7 & 3,89 & 4,04 & 0,030 \\
\hline $25-40$ & 45 & 43,3 & 2,30 & 3,02 & \\
\hline Total & 104 & 100,0 & & & \\
\hline \multicolumn{6}{|l|}{ Estado marital } \\
\hline Casada & 20 & 19,2 & 4,16 & 3,84 & \\
\hline Vive em união & 52 & 50,0 & 2,74 & 2,94 & 0,339 \\
\hline Não vive em união & 32 & 30,8 & 3,35 & 4,61 & \\
\hline Total & 104 & 100,0 & & & \\
\hline \multicolumn{6}{|l|}{ Cor da pele 2} \\
\hline Branca & 23 & 22,3 & 3,13 & 3,23 & \\
\hline Parda & 69 & 67,0 & 3,01 & 3,79 & 0,432 \\
\hline Negra & 11 & 10,7 & 4,57 & 4,21 & \\
\hline Total & 103 & 100,0 & & & \\
\hline \multicolumn{6}{|l|}{ Tabagismo } \\
\hline Fumante & 7 & 6,7 & 1,73 & 5,01 & \\
\hline Ex-fumante & 25 & 24,1 & 4,04 & 3,41 & 0,286 \\
\hline Não fumante & 72 & 69,2 & 3,05 & 3,65 & \\
\hline Total & 104 & 100,0 & & & \\
\hline \multicolumn{6}{|c|}{ Renda familiar per capita (em Reais) } \\
\hline $30,00-299,00$ & 34 & 32,7 & 2,98 & 3,59 & 0,671 \\
\hline $300,00-3400,00$ & 70 & 67,3 & 3,31 & 3,77 & \\
\hline Total & 104 & 100,0 & & & \\
\hline \multicolumn{6}{|l|}{ Escolaridade (anos) } \\
\hline $0-8$ & 43 & 41,3 & 2,67 & 3,32 & 0,224 \\
\hline $9-16$ & 61 & 58,7 & 3,57 & 3,93 & \\
\hline Total & 104 & 100,0 & & & \\
\hline \multicolumn{6}{|l|}{ Estatura (m) } \\
\hline $1,43-1,59$ & 59 & 56,7 & 2,75 & 3,89 & 0,154 \\
\hline $1,60-1,76$ & 45 & 43,3 & 3,80 & 3,39 & \\
\hline Total & 104 & 100,0 & & & \\
\hline \multicolumn{6}{|l|}{ IMC pré-gestacional $\left(\mathrm{kg} / \mathrm{m}^{2}\right)^{3}$} \\
\hline Baixo peso $(<18,5)$ & 5 & 4,8 & 2,40 & 1,82 & \\
\hline Eutrofia $(18,5-24,9)$ & 68 & 65,4 & 3,75 & 3,54 & 0,110 \\
\hline Sobrepeso/ Obesidade $(\geq 25)$ & 31 & 29,8 & 2,11 & 4,07 & \\
\hline Total & 104 & 100,0 & & & \\
\hline \multicolumn{6}{|c|}{ Adequação do ganho de peso total 4} \\
\hline Insuficiente & 37 & 38,5 & 1,45 & 3,08 & \\
\hline Adequado & 33 & 34,4 & 3,63 & 3,03 & $<0,001$ \\
\hline Excessivo & 26 & 27,1 & 5,05 & 3,90 & \\
\hline Total & 96 & 100,0 & & & \\
\hline \multicolumn{6}{|l|}{ Peso ao nascer (g) } \\
\hline $1250-3500$ & 77 & 74,0 & 3,11 & 3,61 & 0,681 \\
\hline $3501-4800$ & 27 & 26,0 & 3,46 & 4,02 & \\
\hline Total & 104 & 100,0 & & & \\
\hline
\end{tabular}

1teste t-Student (comparações entre duas categorias) ou ANOVA (comparações entre três categorias); 2 Um dado faltante para cor de pele; 3 Índice de massa corporal classificado de acordo com o Institute of Medicine (IOM, 2009): 4Ganho de peso gestacional classificado de acordo com o Institute of Medicine (IOM, 2009), 8 dados faltantes. 
Tabela 3

Média de retenção de peso pós-parto segundo as co-variáveis reprodutivas e bioquímicas. Rio de Janeiro, 2005-2007.

\begin{tabular}{|c|c|c|c|c|c|}
\hline Variáveis & N & $\%$ & $\begin{array}{l}\overline{\mathrm{X}} \text { de retenção de peso } \\
\text { pós-parto }(\mathrm{kg})\end{array}$ & DP & $p 1$ \\
\hline \multicolumn{6}{|l|}{ Tipo de parto } \\
\hline Normal & 54 & 52,4 & 3,72 & 3,75 & 0,155 \\
\hline Cesárea & 49 & 47,6 & 2,67 & 3,63 & \\
\hline Total & 103 & 100,0 & & & \\
\hline \multicolumn{6}{|l|}{ Amamentação exclusiva } \\
\hline Sim & 68 & 63,4 & 3,00 & 3,75 & 0,457 \\
\hline Não & 36 & 34,6 & 3,58 & 3,62 & \\
\hline Total & 104 & 100,0 & & & \\
\hline \multicolumn{6}{|l|}{ Idade da menarca (anos) } \\
\hline $8-12$ & 50 & 48,1 & 3,40 & 3,95 & 0,602 \\
\hline $13-18$ & 54 & 51,9 & 3,02 & 3,48 & \\
\hline Total & 104 & 100,0 & & & \\
\hline \multicolumn{6}{|l|}{ Paridade ( $\mathrm{n}^{\circ}$ de partos) } \\
\hline 0 & 60 & 61,5 & 3,75 & 4,31 & 0,077 \\
\hline$\geq 1$ & 44 & 38,5 & 2,45 & 2,51 & \\
\hline Total & 104 & 100,0 & & & \\
\hline \multicolumn{6}{|l|}{ Glicemia (mg/dL) 2} \\
\hline $1^{\circ}$ tercil $(<72,6)$ & 30 & 31,6 & 2,96 & 2,85 & \\
\hline $2^{\circ}$ tercil $(72,6 \leq 80,4)$ & 35 & 36,8 & 3,67 & 4 & 0,588 \\
\hline $3^{\circ}$ tercil $(>80,4)$ & 30 & 31,6 & 2,79 & 4,05 & \\
\hline Total & 95 & 100,0 & & & \\
\hline \multicolumn{6}{|l|}{ Colesterol total $(\mathrm{mg} / \mathrm{dL})^{3}$} \\
\hline $1^{\circ}$ tercil $(<155,0)$ & 27 & 28,7 & 3,60 & 4,55 & \\
\hline $2^{\circ}$ tercil $(155,0 \leq 186,0)$ & 31 & 33,0 & 3,86 & 3,70 & 0,307 \\
\hline $3^{\circ}$ tercil $(>80,4)$ & 36 & 38,3 & 2,51 & 3,21 & \\
\hline Total & 94 & 100,0 & & & \\
\hline \multicolumn{6}{|l|}{ Leptina sérica $(\mathrm{ng} / \mathrm{mL})^{4}$} \\
\hline $1^{\circ}$ tercil $(<12,9)$ & 25 & 33,3 & 3,40 & 3,7 & \\
\hline $2^{\circ}$ tercil $(12,9 \leq 28,5)$ & 23 & 30,7 & 3,06 & 3,33 & 0,840 \\
\hline $3^{\circ}$ tercil $(>28,5)$ & 27 & 36,0 & 2,80 & 3,93 & \\
\hline Total & 75 & 100,0 & & & \\
\hline
\end{tabular}

1 teste t-Student (comparações entre duas categorias) ou ANOVA (comparações entre três categorias); 29 dados faltantes; 310 dados faltantes; 429 dados faltantes. 
Tabela 4

Regressão linear univariada segundo co-variáveis. Rio de Janeiro, 2005-2007.

\begin{tabular}{|c|c|c|c|c|}
\hline Variáveis & $\boldsymbol{\beta}$ & IC $95 \%$ & $\mathbf{R}^{2}$ & $p^{1}$ \\
\hline Idade (anos) & $-0,115$ & {$[-0,24]-0,01$} & 0,032 & 0,069 \\
\hline \multicolumn{5}{|l|}{ Estado marital2 } \\
\hline Não vive em união & $-0,802$ & {$[-2,89]-1,29$} & 0,021 & 0,448 \\
\hline Vive em união & $-1,413$ & {$[-3,34]-0,52$} & & 0,149 \\
\hline \multicolumn{5}{|l|}{ Cor da pele 3} \\
\hline Parda & $-0,132$ & {$[-1,87]-1,61$} & 0,017 & 0,881 \\
\hline Negra & 1,435 & {$[-1,24]-4,11$} & & 0,290 \\
\hline \multicolumn{5}{|l|}{ Tabagismo 4} \\
\hline Ex-fumante & 2,315 & {$[-0,82]-5,45$} & 0,024 & 0,145 \\
\hline Não fumante & 1,324 & {$[-1,57]-4,22$} & & 0,367 \\
\hline Renda familiar per capita (em Reais) & 0,000 & {$[-0,003]-0,002$} & 0,001 & 0,729 \\
\hline Escolaridade (anos) & 0,135 & {$[-0,11]-0,39$} & 0,011 & 0,284 \\
\hline Estatura $(\mathrm{cm})$ & 0,048 & {$[-0,07]-0,16$} & 0,007 & 0,402 \\
\hline IMC pré-gestacional (kg/m²) & $-0,141$ & {$[-0,31]-0,03$} & 0,026 & 0,101 \\
\hline Ganho de peso gestacional total $(\mathrm{kg})$ & 0,486 & $0,36-0,62$ & 0,367 & $<0,001$ \\
\hline Peso ao nascer $(\mathrm{kg})$ & 0,000 & {$[-0,001]-0,002$} & 0,001 & 0,722 \\
\hline Tipo de parto5 & $-1,045$ & {$[-2,50]-0,40$} & 0,020 & 0,155 \\
\hline Tipo de amamentação6 & 0,571 & {$[-0,95]-2,09$} & 0,005 & 0,457 \\
\hline Idade da menarca & $-0,139$ & {$[-0,56]-0,28$} & 0,004 & 0,514 \\
\hline Paridade & $-0,427$ & {$[-1,17]-0,32$} & 0,013 & 0,256 \\
\hline Glicemia (mg/dL) & $-0,005$ & {$[-0,08]-0,07$} & 0,000 & 0,884 \\
\hline Colesterol total (mg/dL) & $-0,016$ & {$[-0,04]-0,01$} & 0,023 & 0,141 \\
\hline Leptina sérica $(\mathrm{ng} / \mathrm{mL})$ & 0,003 & {$[-0,03]-0,04$} & 0,001 & 0,844 \\
\hline
\end{tabular}

1 p refere-se ao Teste F; 2 Categoria de referência = casada; 3 Categoria de referência = brancas; 4 Categoria de referência = fumantes; 5 Categoria de referência $=$ parto normal; 6 Categoria de referência = amamentação exclusiva; IMC= Índice de massa corporal.

\section{Tabela 5}

Modelo final de regressão multivariada. Rio de Janeiro, 2005 - 2007.1

\begin{tabular}{lcccc}
\hline Variáveis & $\boldsymbol{\beta}$ & IC 95\% & $\mathbf{R}^{\mathbf{2}}$ & $\boldsymbol{p}^{\mathbf{2}}$ \\
& & & \\
\hline Ganho de peso gestacional total $(\mathrm{kg})$ & 0,494 & $0,364-0,625$ & $<0,001$ \\
IMC pré-gestacional $\left(\mathrm{kg} / \mathrm{m}^{2}\right)^{3}$ & $-0,149$ & {$[-0,286]-[-0,013]$} & 0,407 & 0,033 \\
Tempo pós-parto (dias) & $-0,016$ & {$[-0,035]-0,004$} & 0,114
\end{tabular}

1 Modelo final $(\mathrm{N}=96) ;{ }^{2}$ p refere-se ao teste $\mathrm{F} ;{ }^{3} \mathrm{IMC}=$ Índice de massa corporal. 


\section{Discussão}

Os resultados observados no presente estudo revelaram que o ganho de peso gestacional encontra-se positivamente associado com a retenção de peso pósparto e inversamente associado ao IMC pré-gestacional e à idade. As mulheres mais jovens apresentaram maiores médias de retenção de peso, porém essa variável apresentou significância limítrofe no modelo multivariado. Não foram observadas associações entre variáveis bioquímicas e retenção de peso pós-parto.

Os principais fatores envolvidos na variação de peso no pós-parto e, consequentemente, no desenvolvimento da obesidade são: ganho de peso gestacional,3,7,11 IMC pré-gestacional,11,12 dieta, 13 aleitamento materno, 14,15 atividade física, $11,13 \mathrm{e}$ fatores sócio-demográficos como idade, 6 escolaridade, renda, ${ }^{16}$ paridade 3,11 e raça. ${ }^{17}$ Esses resultados foram sistematizados por Castro et al.,18 em recente revisão de literatura.

O presente estudo chama atenção para o fato de que aproximadamente $30 \%$ das mulheres apresentam valores de retenção de peso pós-parto com 55 dias em torno de $5 \mathrm{~kg}$, próximo ao observado por Olson et al. 19 e bem superior aos $14 \%$ observados por Ohlin e Rössner 20 em mulheres um ano após o parto. Esses resultados confirmam a hipótese de que o ciclo reprodutivo é um período de risco para a mulher desenvolver sobrepeso e obesidade. 4 As comparações devem considerar o tempo transcorrido desde o parto, mas independente do mesmo, trata-se de um problema relevante de saúde pública.

A proporção de mulheres com ganho de peso gestacional dentro das recomendações observada no presente estudo de $30 \%$ é similar ao reportado por Olson et al. ${ }^{19}$ e por outras investigações. ${ }^{21}$ Por ser um fator passível de intervenção, o ganho de peso gestacional merece especial atenção. Lacerda e Leal ${ }^{8}$ observaram em uma revisão sistemática que dez de onze estudos encontraram associação positiva entre ganho de peso gestacional e retenção de peso pósparto. A influência do IMC pré-gestacional sobre a retenção de peso pós-parto ainda carece de acúmulo de evidências e os resultados têm se mostrado contraditórios. Enquanto alguns estudos não encontraram associação entre IMC pré-gestacional e retenção de peso pós-parto, 22 outros encontraram associação negativa, ${ }^{2,23}$ ou revelaram aumento da retenção pós-parto com o aumento do IMC prégestacional. 24,25

No presente estudo as mulheres mais jovens e as primigestas apresentaram maiores retenções de peso. A idade materna e a paridade são dois importantes fatores determinantes da retenção de peso pós-parto. Aproximadamente $60 \%$ da amostra foi composta por primigestas jovens, com menos de 24 anos. Esses resultados corroboram com outros citados na literatura.6,26 Geralmente, mulheres mais jovens têm IMC menor6 e ganham mais peso durante a gestação, assim como primigestas tendem a ganhar mais peso do que multíparas. 6,26 No estudo longitudinal realizado por Scholl et al. 27 com 274 mulheres americanas que tinham IMC pré-gestacional normal, a proporção de mulheres com ganho de peso considerado excessivo foi maior entre as mais jovens. $\mathrm{O}$ mesmo ocorreu entre as primigestas. ${ }^{27}$ A primeira gestação parece determinar a maior mudança na composição corporal, principalmente em mulheres com excesso de peso. 28

Como limitação do estudo a perda de seguimento de $60 \%$ pode ter subestimado o efeito das associações das variáveis independentes com o desfecho. As perdas de seguimento consistem em um problema inerente aos estudos longitudinais e são difíceis de serem evitadas.

Embora tenha havido variabilidade na aferição do desfecho, é provável que esta aferição em media de 55 dias pós-parto não tenha interferido nos resultados, pois o tempo pós-parto foi incluído no modelo final como variável de controle. O tempo de seguimento, a definição da variável "amamentação" e as perdas de seguimento podem ter subestimado o efeito da associação desta variável com a retenção de peso, já que outros estudos observaram efeito protetor dessa variável.14,29 Outra questão metodológica que merece ser destacada é a provável subestimação do ganho de peso no período gestacional e consequentemente a retenção de peso pósparto, visto que o ganho de peso gestacional foi calculado com base em dados da quarta onda de seguimento, realizada em média com 36 semanas gestacional.

Em resumo, observou-se no presente estudo que o IMC pré-gestacional associou-se inversamente e o ganho de peso gestacional positivamente com o desenvolvimento da retenção de peso pós-parto. A incidência de retenção de peso pós-parto é elevada e deve configurar como indicador a ser monitorado. $\mathrm{O}$ adequado acompanhamento nutricional da gestante pode evitar a ocorrência de diversos desfechos adversos. 


\section{Referências}

1. York DA, Rossner S, Caterson I, Chen CM, James WPT, Kumanyika S, Martorell R, Vorster HH, American Heart Association. Obesity, a worldwide related to heart disease and stroke group I: worldwide demographics of obesity. Circulation. 2004; 110: e463-70.

2. Huang TT, Wang HS, Dai FT. Effect of pre-pregnancy body size on postpartum weight retention. Midwifery. 2010; 26: 222-31. doi:10.1016/j.midw.2008.05.001.

3. Gunderson EP, Abrams B. Epidemiology of gestational weight gain and body weight changes after pregnancy. Epidemiol Rev. 1999; 22: 261-74.

4. Linné Y, Barkeling B, Rössner S. Long term weight development after pregnancy. Obes Rev. 2002; 3: 75-83.

5. Nuss H, Freeland-Graves J, Clarke K, Klohe-Lehman D, Milani TJ. Greater nutrition knowledge is associated with lower 1-year postpartum weight retention in low-income women. J Am Diet Assoc. 2007; 107: 1801-6.

6. Rosenberg L, Palmer JR, Wise LA, Horton NJ, Kumanyika SK, Adams-Campbell LL. A prospective study of the effect of childbearing on weight gain in african-american women. Obes Res. 2003; 11: 1526-35.

7. Linné Y, Dye L, Barkeling B, Rössner S. Long term weight development in women: A 15-year follow-up of the effects of pregnancy. Obes Res. 2004; 12: 1166-78.

8. Lacerda MEA, Leal MC. Fatores associados com a retenção e o ganho de peso pós-parto: uma revisão sistemática. Rev Bras Epidemiol. 2004; 7: 187-200.

9. Institute of Medicine; National Research Council. Weight Gain During Pregnancy: Reexamining the Guidelines. Washington (DC): National Academy of Science; 2009.

10. Gordon CC, Chumlea WC, Roche AP. Stature, recumbent length, and weight. In: Lohman TG, Roche AF, Martorel R, editor. Anthropometric standardization reference manual. Champaign, Illinois: Human Kinetics Books; 1988. p. 3954.

11. Boardley DJ, Sargent RG, Coker AL, Hussey JR, Sharpe PA. The relationship between diet activity and other factors and postpartum weight change by race. Obstet Gynecol. 1995; 86: 834-8.

12. Siega-Riz AM, Laraia B. The implications of maternal overweight and obesity on the course of pregnancy and birth outcomes. Matern Child Health. J 2006; 10: S153-6.

13. Amorim AR, Rössner S, Neovius M, Lorenço PM, Linné Y. Does excess pregnancy weight gain constitute a major risk for increasing long-term BMI? Obes Res. 2007; 15: 127886

14. Kac G, Benício MHD, Velásquez-Meléndez G, Valente JG. Nine months postpartum weight retention predictors for Brazilian women. Public Health Nutr. 2004; 75: 621-8.

15. Rooney BL, Schauberger CW. Excess pregnancy weight gain and long-term obesity: one decade later. Obstet Gynecol. 2002; 100: 245-52.
16. Shrewsbury VA, Robb KA, Power C, Wardle J Socioeconomic differences in weight retention, weightrelated attitudes and practices in postpartum women. Matern Child Health J. 2009; 13: 231-40.

17. Keppel KG, Taffel SM. Pregnancy-related weight gain and retention: implications of the 1990 Institute of Medicine Guidelines. Am J Public Health. 1995; 83: 1100-3.

18. Castro MBT, Kac G, Sichieri R. Determinantes nutricionais e sócio-demográficos da variação de peso no pós-parto: uma revisão da literatura. Rev Bras Saúde Matern Infant. 2009; 9: 125-37.

19. Olson CM, Strawderman MS, Hinton PS, Pearson TA Gestational weight gain and postpartum behaviors associated with weight change from early pregnancy to $1 \mathrm{y}$ postpartum. Int J Obes. 2003; 27: 117-27.

20. Öhlin A, Rössner S. Trends in eating patterns, physical activity and socio-demographic factors I relation to postpartum body weight development. Br J Nutr. 1994; 71: 457 70 .

21. Nucci LB, Duncan BB, Mengue SS, Branchtein L, Schmidt MI, Fleck ET. Assessment of weight gain during pregnancy in general prenatal care services in Brazil. Cad Saúde Pública. 2001; 17: 1367-74.

22. Valeggia CR, Ellison PT. Impact of breastfeeding on anthropometric changes in peri-urban Toba women (Argentina). Am J Human Biol. 2003; 15: 717-24.

23. Martinez H, Allen LH, Lung'aho M, Chavez A, Pelto GH. Maternal fatness in Mexican women predicts body composition changes in pregnancy and lactation. Adv Exp Med Biol. 1994; 352: 99-107.

24. Coitinho DC, Sichieri R, D'Aquino Benicio MH. Obesity and weight change related to parity and breast-feeding among parous women in Brazil. Public Health Nutr. 2001; 4: 865-70.

25. Sichieri R, Field AE, Rich-Edwards J, Willet WC. Prospective assessment of exclusive breastfeeding in relation to weight change in women. Int J Obes. 2003; 27: 81520 .

26. Stulbach TE, Benício MHA, Andreazza R, Kono S Determinantes do ganho ponderal excessivo durante a gestação em serviço público de pré-natal de baixo risco. Rev Bras Epidemiol. 2007; 10: 99-108.

27. Scholl TO, Hediger ML, Schall JI, Ances IG, Smith WK. Gestacional weight gain, pregnancy outcome, and postpartum weight retention. Obstet Gynecol. 1995; 86: 423-7.

28. Gunderson EP, Murtaugh MA, Lewis CE, Quesenberry CP, West DS, Sidney S. Excess gains in weight and waist circumference associated with childbearing: the coronary artery risk development in young adults study (CARDIA). Int J Obes. 2004; 28: 525-35.

29. Janney CA, Zhang D, Sowers M. Lactation and weight retention. Am J Clin Nutr.1997; 66:1116-24.

Recebido em 1 de junho de 2009

Versão final apresentada em 29 de abril de 2010

Aprovado em 10 de maio de 2010 